\title{
Novo registro para Litopeltis Hebard, 1920 no Brasil e descrição de duas espécies novas (Blaberidae, Epilamprinae)
}

\author{
Sonia Maria Lopes ${ }^{1,2}$ \& Edivar Heeren de Oliveira ${ }^{1}$ \\ ${ }^{1}$ Departamento de Entomologia, Museu Nacional, Universidade Federal do Rio de Janeiro - UFRJ, \\ Quinta da Boa Vista, São Cristóvão, CEP 20940-040, Rio de Janeiro, RJ, Brasil \\ ${ }^{2}$ Autor para correspondência: Sonia Maria Lopes, e-mail: sonialfraga@gmail.com
}

LOPES, S.M. \& OLIVEIRA, E.H. New records of Litopeltis Hebard, 1920 from Brazil and description of two new species (Blaberidae, Epilamprinae). Biota Neotrop. 10(2): http://www.biotaneotropica.org.br/v10n2/ en/abstract?article+bn01910022010.

Abstract: In this paper was assinalated one new records and two new species of Litopeltis from Brazil: L. paineirense sp. nov and L. ribeiropretano sp. nov. which are similar to L. bispinosa (Saussure, 1893) and distinguished by characters of the morphology of genitalia.

Keywords: Rio de Janeiro, São Paulo, Blattaria, taxonomy.

LOPES, S.M. \& OLIVEIRA, E.H. Novo registro para Litopeltis Hebard, 1920 no Brasil e descrição de duas espécies novas (Blaberidae, Epilamprinae). Biota Neotrop. 10(2): http://www.biotaneotropica.org.br/v10n2/ pt/abstract?article+bn01910022010.

Resumo: Neste trabalho é assinalado o novo registro e a descrição de duas espécies novas de Litopeltis para o Brasil: L. paineirense sp. nov e L. ribeiropretano sp. nov., as quais são similares à L. bispinosa (Saussure, 1893) e diferenciam-se por caracteres de morfologia de genitália.

Palavras-chave: Rio de Janeiro, São Paulo, Blattaria, taxonomia. 


\section{Introdução}

O gênero Litopeltis foi descrito por Hebard (1920) com base em material procedente da Colômbia sendo incluído na subfamília Perisphaerinae. Este gênero está relacionado a dois gêneros: Colapteroblatta Hebard, 1920 e Mioblatta Saussure, 1895. Diferindo do último pelo tamanho, ausência de pubescência, vértice liso e sem carena, pronoto sem manchas, margem cefálica apresentando-se fracamente truncada, asas com campo marginal mais estreito, a veia ulnar com ramos incompletos e numerosos; e fêmures espinhosos. Quanto à Colapteroblatta, Litopeltis é próximo em relação à forma e disposição de veias nas tégminas e asas, e a distribuição dos espinhos nas pernas; porém difere pelo tamanho total e ocelos pouco definidos. A espécie-tipo designada foi L. bispinosa (Saussure), anteriormente considerada Calolampra bispinosa Saussure, 1893.

Rehn (1928) considerou Litopeltis similar à Phoetalia Shelford, 1910 (antigo Leurolestes Rehn \& Hebard, 1914) pelo aspecto geral, e pela configuração das placas subgenitais, ambas assimétricas, projetadas medianamente, com os estilos finos inseridos lateralmente, porém o exame da espinhação do fêmur anterior de Phoetalia nos permitiu separá-los de Litopeltis, pois naquele gênero os espinhos se dispõem também em série, porém de espinhos diminutos podendo apresentar de um a cinco outros espinhos robustos dispostos entre eles.

Princis (1960), Mckittrick (1964) e Roth (1970), contrariando Hebard, colocaram o gênero entre os Blaberidae, porém somente Mckittrick (1964) colocou-o em Epilamprinae. Os outros dois autores o posicionaram em Laxtinae. Princis (1963) listou 8 espécies para o gênero, sendo uma do Panamá e 7 de Costa Rica.

Roth (1971), com base na ovoviviparidade e pela configuração do L2d e o prepúcio na genitália masculina, colocou-os na tribo Epilamprini. Assinalou também serem as genitálias de Litopeltis e Cariacasia Rehn, 1928 muito similares e afastou Litopeltis de Colapteroblatta colocando em Poroblattini e de Phoetalia que foi colocado em Blaberinae.

Rocha \& Silva (1982) reportou três exemplares machos da localidade de Paineiras (RJ) como Phoetalia pallida (Brunner, 1865).

Roth \& Gutierrez (1998) acrescentaram mais uma espécie ao gênero ampliando a distribuição geográfica até o Equador.

Beccalloni (2007) considerou nove espécies para o gênero reafirmando a presença do mesmo na América Central (Costa Rica e Panamá) e na América do Sul (Equador) e Vélez (2008) ratificou a presença do gênero na Colômbia.

Examinando o material estudado por Rocha \& Silva (1982), foi verificado em relação à espinhação de pernas e configuração da genitália, que os exemplares apresentam caracteres de Litopeltis. Isso levou-nos a retirá-los de Phoetalia e descrevê-los como espécies novas de Litopeltis, caracterizando o primeiro registro do gênero para o Brasil.

\section{Material e Métodos}

As genitálias dos espécimens foram dissecadas e devidamente preparadas e examinadas em lâminas com glicerina. A metodologia segue Lopes \& Oliveira (2000). A terminologia adotada para genitália foi baseada em Roth (2003). Todo o material encontra-se depositado na coleção do Departamento de Entomologia do Museu Nacional (MNRJ).

\section{Resultados}

\section{Litopeltis paineirense sp. nov.}

Coloração geral: castanho-clara brilhosa. Cabeça com vértice, espaço interocular, fronte e região centro-basal castanhos; palpos com tomentosidade dourada. Antenas com os dez primeiros artículos brilhosos, os demais foscos e muito tomentosos. Pronoto com mancha central, ampla, castanho mais escuro. Pernas com a base das coxas, espinhos das tíbias, parte dorsal dos tarsos e unhas castanho-escuros. Pulvilos e arólios esbranquiçados. Tégminas com o tronco inicial de todas as veias castanhoescuros, apresentando no setor discoidal, veias transversais com pigmentação mais evidenciada. Abdome castanho-escuro, com margens laterais dos esternitos castanho-claras amareladas.

Dimensões (mm): Holótipo $\widehat{\sigma}$ - comprimento total: 25,0; comprimento do pronoto: 4,5 ; largura do pronoto: 6,5 ; comprimento da tégmina: 22,0; largura da tégmina: 6,0 .

Tamanho grande para o gênero. Cabeça com o vértice parcialmente exposto; espaço interocular amplo subigual à área que separa as bases das inserções antenais Antenas tomentosas, não alcançando o ápice do abdome. Ocelos desenvolvidos. Palpos maxilares tomentosos e desenvolvidos, primeiro e segundo artículos pequenos, quarto artículo levemente menor que o terceiro, quinto artículo dilatado, intensamente tomentoso e maior que os demais (Figura 1a).

Tórax. Pronoto transverso, trapezoidal, convexo, de ápice quase reto e base levemente angular; abas laterais amplas e arredondadas (Figura 1b). Pernas desenvolvidas e robustas. Fêmur I na face ântero-ventral apresentando uma série de sete espinhos finos, da base até próximo à região mediana do fêmur, espaçados e subiguais, seguidos por uma série de 11 pequenos e subiguais, mais aproximados uns dos outros, terminando por um espinho apical grande e forte; face póstero-ventral com quatro espinhos espaçados, sendo um apical forte, precedido por um pequeno e robusto espinho. Fêmures II e III na face ântero-ventral com dois espinhos pequenos e robustos, sendo um na região mediana e outro apical; face póstero-ventral com três a cinco espinhos robustos espaçados, sendo um deles apical. Tarsos desenvolvidos e tomentosos. Pulvilos pequenos e presentes em todos os artículos tarsais; arólios presentes, porém pequenos. Unhas simétricas com superfície ventral na borda com pequenas ondulações. Tégmina longa ultrapassando o ápice dos cercos, mais larga entre a região mediana e o terço apical; campo marginal curto, estreito e levemente côncavo, campo escapular longo e estreito com disposição oblíqua das veias; campo discoidal convexo, amplo, com disposição longitudinal, apresentando veias transversais bem marcadas; campo anal amplo, convexo com seis ou sete veias axilares. Asas com setor costal apresentando veias simples; triângulo apical não visível; campo anal dobrado em leque.

Abdome: ausência de modificação tergal. Placa supraanal arredondada com reentrância mediana pequena; cercos curtos; paraproctos evidenciados e afilados (Figura 1c). Placa subgenital típica de blaberídeo, com leve proeminência mediana; estilos afilados (Figura 1d). Falômero esquerdo esclerotinizado medianamente (Figura 1e). Esclerito mediano desenvolvido com o ápice curto sem forma definida (Figura 1f). Falômero direito apresentando forma de gancho apicalmente e com um espinho pré-apical (Figura $1 \mathrm{~g}$ ), que o diferencia das demais espécies.

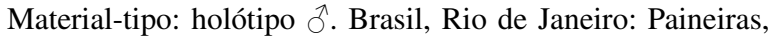
XI/1953, Thomaz, Machado e Rego Barros cols .

Etimologia: O nome é alusivo à localidade de coleta do holótipo.

Comentário: Espécie similar à L. bispinosa (Saussure, 1893) diferindo por caracteres da morfologia da genitália descritos acima. 

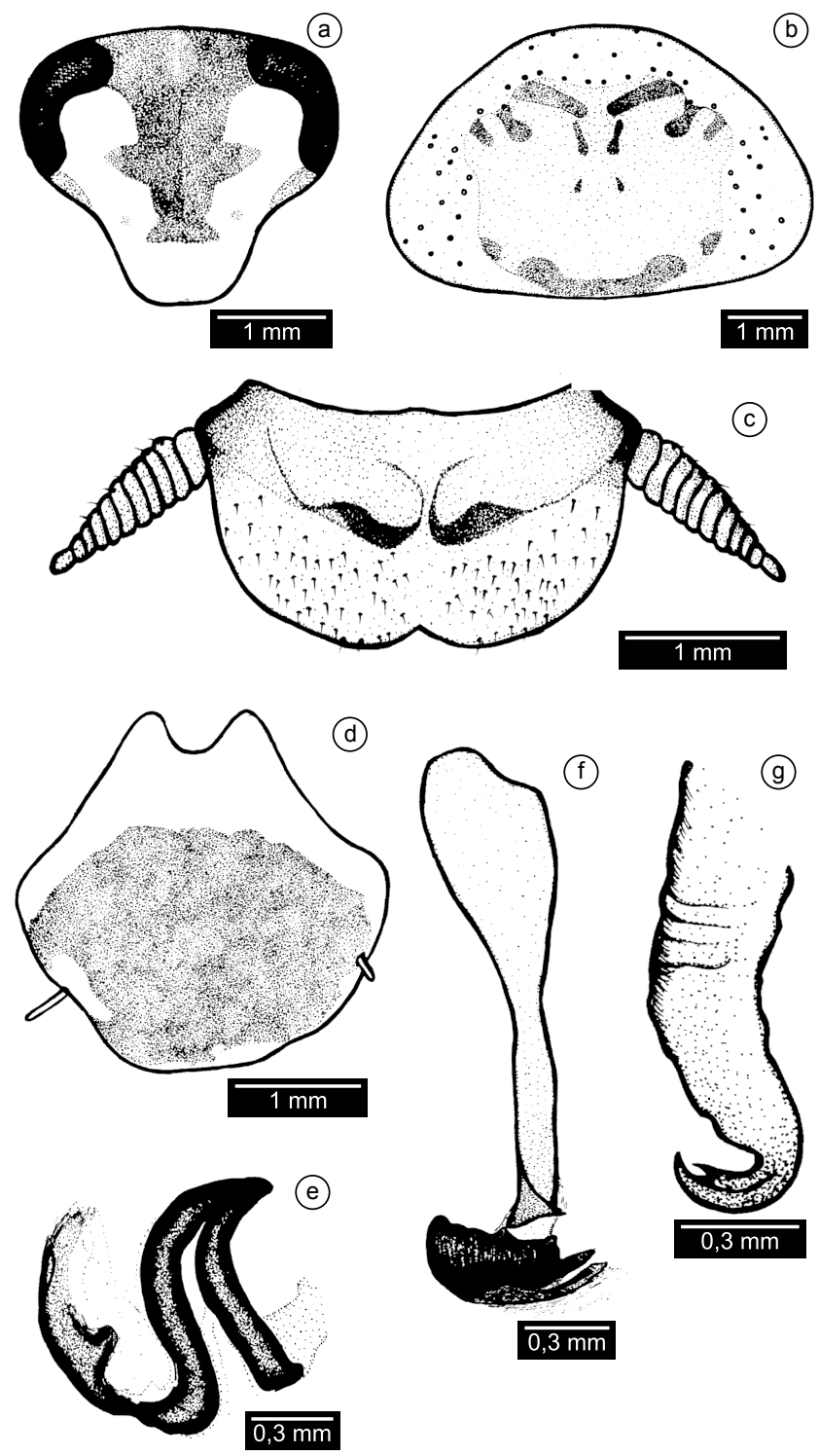

Figura 1. Litopeltis paineirense sp. nov., holótipo $\widehat{\jmath}$. a) cabeça; b) pronoto; c) placa supra-anal, d) placa subgenital; e) falômero esquerdo; f) esclerito mediano; g) falômero direito.

Figure 1. Litopeltis paineirense sp. nov., holotype $\widehat{\delta}$. a) head; b) pronotum; c) supra-anal plate, d) subgenital plate; e) left phallomere; f) median sclerite; g) right phallomere.

\section{Litopeltis ribeiropretano sp. nov.}

Coloração geral: castanho-clara brilhosa. Cabeça com olhos escuros, vértice, espaço interocular, fronte e base do clípeo castanhos; antenas com os oito primeiros artículos brilhosos e os demais tomentosos e foscos. Pronoto com mancha central com formato irregular em castanho mais escuro, com nuances castanhas quase negras. Pernas com a base das coxas, espinhos das tíbias, parte dorsal dos tarsos e unhas mais pigmentados. Pulvilos e arólios esbranquiçados. Tégminas com o tronco inicial de todas as veias mais escuro, apresentando no setor discoidal, veias transversais com pigmentação pouco evidenciada. Abdome castanho-escuro, ventralmente apresentando as margens laterais castanho-claras amareladas.
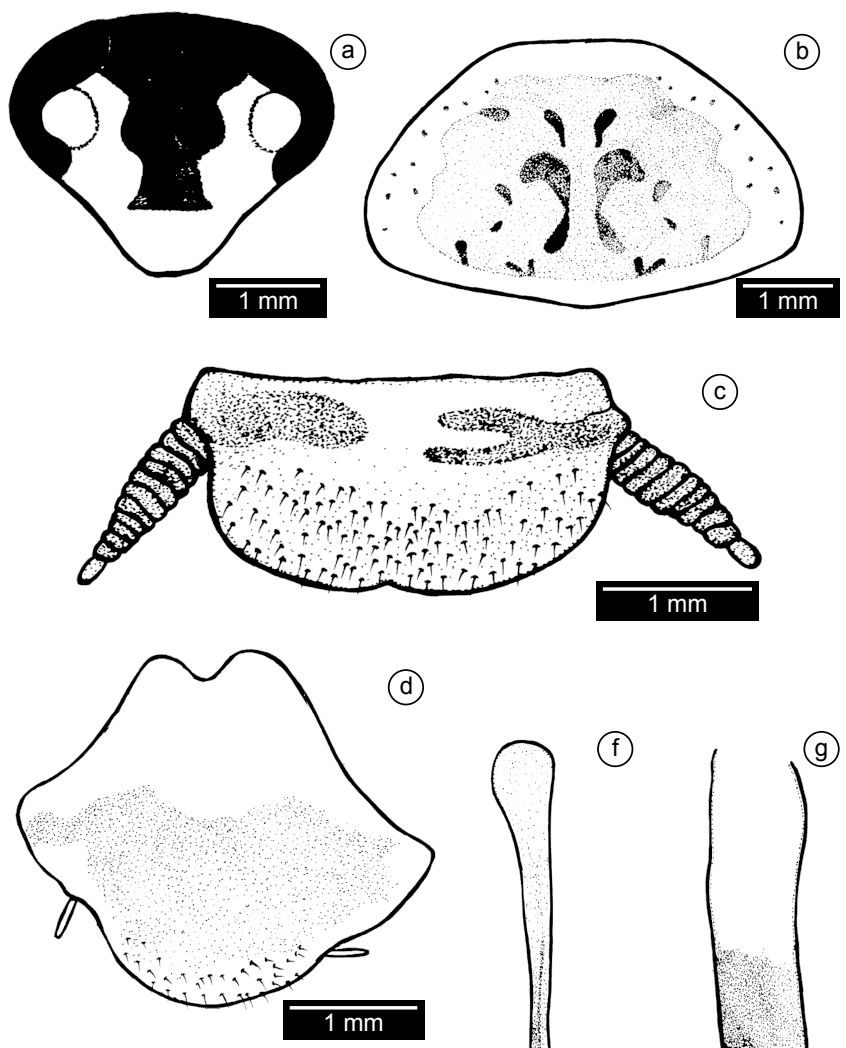

(d)
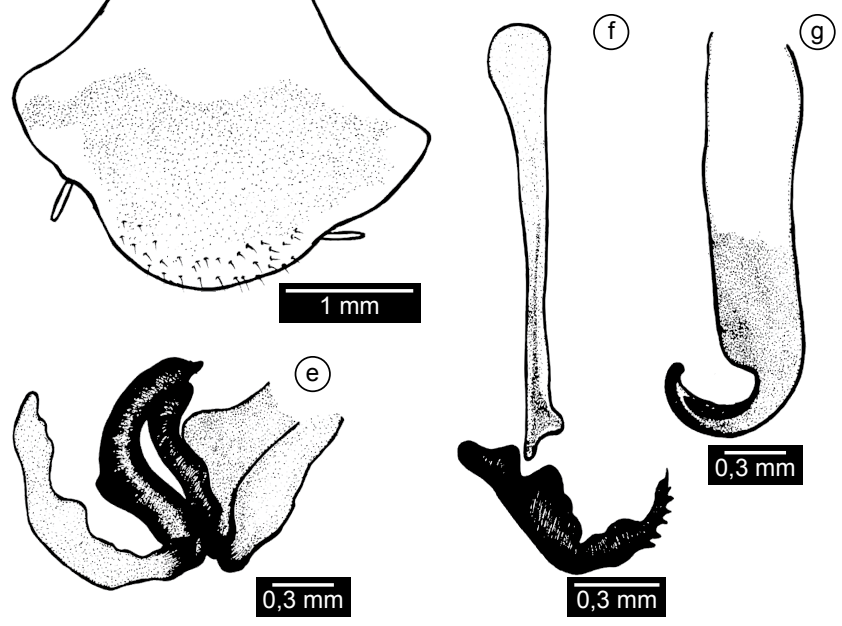

Figura 2. Litopeltis ribeiropretano sp. nov., holótipo $\widehat{\jmath}$. a) cabeça; b) pronoto; c) placa supra-anal; d) placa subgenital; e) falômero esquerdo; f) esclerito mediano; g) falômero direito.

Figure 2. Litopeltis ribeiropretano sp. nov., holotype $\lesssim$. a) head; b) pronotum; c) supra anal plate; d) subgenital plate; e) left phallomere; f) median sclerite; g) right phallomere.

Dimensões (mm): Holótipo đ comprimento total: 20,0; comprimento do pronoto: 3,5 ; largura do pronoto: 5,0 ; comprimento da tégmina: 17,0; largura da tégmina: 5,0.

Cabeça: vértice parcialmente exposto; espaço interocular amplo subigual à área que separa as bases das inserções das antenas (Figura 2a). Antenas tomentosas, alcançando pouco mais da metade do comprimento do abdome. Ocelos desenvolvidos. Palpos maxilares tomentosos e desenvolvidos, terceiro e quarto artículos subiguais em tamanho, quinto artículo dilatado maior que os anteriores.

Tórax. pronoto transverso, trapezoidal, convexo, de ápice quase reto e base levemente angular; abas laterais amplas e arredondadas (Figura 2b). Pernas pouco longas e robustas. Fêmur I na face ântero-ventral apresentando uma série de 3-5 espinhos finos e subiguais, espaçados medianamente, seguidos por série de espinhos de 18-22 espinhos pequenos, subiguais, mais próximos uns aos outros, terminando por um apical longo e forte; face póstero-ventral com espinhos finos e 
espaçados, terminando por um apical forte e menor que o apical da face anterior. Fêmures II e III, na face ântero-ventral com dois espinhos, sendo um mediano e um apical, ambos pequenos e robustos; face póstero-ventral com três a cinco espinhos espaçados, sendo um deles apical. Tarsos desenvolvidos e tomentosos; perna III, com artículo basal do tarso longo, igual ou maior em tamanho aos demais artículos, o primeiro artículo apresenta ventralmente duas fileiras de espinhos pequenos, ao longo de todo o artículo. Pulvilos grandes e presentes em todos os artículos tarsais; arólios presentes e pequenos; unhas simétricas e com sutil e pouco notada especialização ventral. Tégmina longa, ultrapassando o ápice dos cercos, mais larga entre a região mediana o terço apical, estreitando em direção ao ápice; campo marginal côncavo e bem marcado; campo escapular estreito e longo com disposição oblíqua das veias apicalmente; campo discoidal convexo amplo, alargado apicalmente com disposição longitudinal das veias e o campo anal amplo, alongado com 10 veias axilares. Asas com setor costal com ápices das veias não dilatadas; triângulo apical não visível; campo anal dobrado em leque.

Abdome: modificação tergal ausente. Placa supra-anal ciliada, curta e alargada com reentrância mediana pouco perceptível; cercos curtos (Figura 2c). Placa subgenital alargada e proeminente medianamente, estilos afilados na região médio apical da placa (Figura 2d). Falômero direito em forma de gancho apicalmente com reentrância acentuada próximo ao ápice (Figura $2 \mathrm{~g}$ ); falômero esquerdo com estrutura mediana esclerotinizada (Figura 2e); esclerito mediano desenvolvido apresentando o ápice esclerotinizado sem forma definida com projeções espiniformes (Figura 2f).

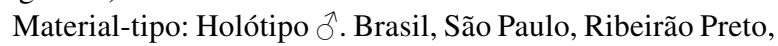
Fazenda de Pedra, 1/VI/1954, Travassos col.; Parátipo $\widehat{\sigma}$. Mato Grosso, Sinop, $12^{\circ} 31^{\prime} \mathrm{S}$ e $55^{\circ} 37^{\prime} \mathrm{W}, \mathrm{Br}-163$, km 500-600, $350 \mathrm{~m}$ de altitude; Parátipo $\widehat{\delta}$. Rio de Janeiro, Itatiaia, Lago Azul, 18-21/VI/1955, R.S.-Albuquerque \& equipe col., $\mathrm{n}^{\circ} 274$ (MNRJ).

Etimologia: O nome é alusivo à localidade de coleta do holótipo.

Diagnose: Espécie próxima a L. bispinosa (Saussure, 1893), dela diferenciando-se por caracteres de genitália do macho e distintamente a posição dos espinhos do ápice do esclerito mediano.

\section{Agradecimentos}

À FAPERJ, projeto "Preservação e guarda do acervo do Departamento de Entomologia do Museu Nacional/UFRJ, pelo apoio logístico.

\section{Referências Bibliográficas}

BECCALONI, G.W. 2007. Blattodea Species File Online. Version 1.2/3.4. http://blattodea.speciesdile.org (último acesso em 10/10/2008).

HEBARD, M. 1920. The Blattidae of Panama. Memoirs of the Entomological Society $4: 1-148$.

LOPES, S.M. \& OLIVEIRA, E.H. 2000. Espécie nova de Eublaberus Hebard, 1919 do Estado de Goiás, Brasil e notas sobre E. marajoara Rocha e Silva-Albuquerque, 1972 (Blaberidae, Blaberinae). Boletim do Museu Nacional Zoologia 433:1-5.

MCKITTRICK, F.A. 1964. Evolutionary studies of cockroaches. Memoir Cornell University Agricultural Experiment Station. 389:1-197.

PRINCIS, K. 1960. Zur Systematik der Blattarien. Eos. Revista Espanola de Entomologia 36:427-449.

PRINCIS, K. 1963. Blattariae: Subordo Polyphagoidea: Fam.: Homeogamiidae, Euthyrraphidae, Latindiidae, Anacompsidae, Atticolidae, Attaphilidae. Subordo Blaberoidea: Fam. Blaberidae. In Orthopterorum Catalogus (M. Beier, ed.). Uitgeverij Dr. W. Junk: s', Gravenhage, pars 4, p. 76-172.

REHN, J.A.G. 1928. New or little known Neotropical Blattidae (Orthoptera). Transactions of the American Entomological Society 54:125-194.

ROCHA E SILVA, I. 1982. Lista dos Blattodea do Município do Rio de Janeiro, RJ, Brasil (Dictyoptera). Boletim do Museu Nacional Zoologia 304:1-20.

ROTH, L.M. 1970. The male genitália of Blattaria. IV. Blaberidae: Blaberinae. Psyche 77:217-236.

ROTH, L.M. 1971. The male genitalia of Blattaria. VII. Galiblatta, Dryadoblatta, Parcoblatta, Colapteroblatta, Nauclidas, Notolampra, Litopeltis and Caricasia (Blaberidae: Epilamprinae). Psyche 8:180-192.

ROTH, L.M. 2003. Systematic and Phylogeny of Cockroaches (Dictyoptera: Blattaria). Orient. Insec. 37:1-186

ROTH, L.M. \& GUTIERREZ, E. 1998. The cockroach genus Colapteroblatta, its synonyms Poroblatta, Acroporoblatta, and Nauclidas, and a new species of Litopeltis (Blattaria: Blaberidae, Epilamprinae). T. Am. Entomol. Soc. 124(3-4):167-202.

VÉLEZ, A. 2008. Checklist of Colombian cockroaches (Dictyoptera, Blattaria). Biota Colomb. 9(1):21-38.

Recebido em 21/12/09

Versão reformulada recebida em 08/05/10

Publicado em 10/05/10 